\title{
Power Aware Mobility Management of M2M for IoT Communications
}

\author{
Awais Ahmad, ${ }^{1}$ Anand Paul, ${ }^{1}$ M. Mazhar Rathore, ${ }^{1}$ and Seungmin Rho $^{2}$ \\ ${ }^{1}$ School of Computer Science and Engineering, Kyungpook National University, Daegu 702-701, Republic of Korea \\ ${ }^{2}$ Department of Multimedia, Sungkyul University, Anyang 431-003, Republic of Korea
}

Correspondence should be addressed to Anand Paul; paul.editor@gmail.com

Received 28 May 2015; Accepted 29 July 2015

Academic Editor: Neeraj Kumar

Copyright (C) 2015 Awais Ahmad et al. This is an open access article distributed under the Creative Commons Attribution License, which permits unrestricted use, distribution, and reproduction in any medium, provided the original work is properly cited.

Machine-to-Machine (M2M) communications framework is evolving to sustain faster networks with the potential to connect millions of devices in the following years. M2M is one of the essential competences for implementing Internet of Things (IoT). Therefore, various organizations are now focusing on enhancing improvements into their standards to support M2M communications. Thus, Heterogeneous Mobile Ad Hoc Network (HetMANET) can normally be considered appropriate for M2M challenges. These challenges incorporated when a mobile node (MN) selects a target network in an energy efficient scanning for efficient handover. Therefore, to cope with these constraints, we proposed a vertical handover scheme for handover triggering and selection of an appropriate network. The proposed scheme is composed of two phases. Firstly, the MNs perform handover triggering based on the optimization of the Receive Signal Strength (RSS) from an access point/base station (AP/BS). Secondly, the network selection process is performed by considering the cost and energy consumption of a particular application during handover. Moreover, if there are more networks available, then the MN selects the one provided with the highest quality of service (QoS). The decision regarding the selection of available networks is made on three metrics, that is, cost, energy, and data rate. Furthermore, the selection of an AP/BS of the selected network is made on five parameters: delay, jitter, Bit Error Rate (BER), communication cost, and response time. The numerical and experimental results are compared in the context of energy consumption by an MN, traffic management on an AP/BS, and QoS of the available networks. The proposed scheme efficiently optimizes the handoff related parameters, and it shows significant improvement in the existing models used for similar purpose.

\section{Introduction}

Adaptation of heterogeneous access network and efficient use of available resources are one of the key confront for the next generation of mobile communication. M2M practices miscellaneous models in terms of the electronic smart grid, connected cars, body area network, and Android communication using vehicular technologies [1]. M2M communication is an important facilitating technology for Internet of Things (IoT), which facilitates an outsized number of implicated escorts to a large number of research challenges [2]. In 3GPP terminology, $\mathrm{M} 2 \mathrm{M}$ communication is usually referred to as Machine-Type Communication (MTC) [3-5].

Over the last few years, the numbers of heterogeneous networks available at a particular location were increased noticeably [6]. Various communication networks show inherent characteristics in terms of handover failure and consumption of energy and cost, which offer higher communication performance. To highlight the eventual function of M2M communication for developing wide-ranged connections among various devices, the potential of HetMANET cannot be neglected in this regard.

A handover process starts from the machine when it experiences a weak received RSS from a base BS/AP. When RSSI reached a predefined threshold, machine (MN) starts to search for available networks. The handover time is mainly dependent on the scanning delay of the available networks. Furthermore, an optimum network can be selected for effective handover process among available networks on the basis of price, security, transmission rate, and quality of service (QoS). 
Employing the available technologies for MTC leads to various challenges, including a selection of the best network for handover, incompatibility among different networks, and handover delay. To address these challenges in the HetMANET, an efficient, organized handover management scheme is required, which can switch communication data from one network to another with the minimum packet loss and delay, respectively. When a device is moving from one $\mathrm{BS} / \mathrm{AP}$ to another, it executes a discovery mechanism for searching nearby BSs/APs and then establishes a connection with higher QoS. The selection of inappropriate network introduces high handover time and delay in a handover process. This handover delay can be minimized by adjusting different factors like RSS, data rate, available bandwidth, and Signal to Interference and Noise Ratio (SINR) from a BS $[7,8]$.

In 2008, International Telecommunication Union-Radio Sector (ITU-R) defined new specifications for $4 \mathrm{G}$ standard called International Mobile Telecommunication Advanced (IMT-Advanced). IMT-Advanced supports $100 \mathrm{Mbits} / \mathrm{s}$ for high mobility connections and $1 \mathrm{Gbits} / \mathrm{s}$ for low mobility connections [9]. With the increase in data rates, new technologies such as WiFi and WiMAX participate actively to develop new technology modification. Development of new technologies is urgently needed since these networks face key constraints of compatibility issues.

The IEEE 802.21 is the MIH standard in 2008 for seamless handover between networks of the same and different type [10]. Recently, much research has been performed to improve the currently available MIH standard [11-13]. The MIH standard is still facing many challenges that need to be addressed; that is, (i) long handover time is required for the MIIS server, which is located many hops away, (ii) time needed for handover process is very short when the handover number is frequent in a handover region, and (iii) failure of a hop requires alternate routes to connect MIIS server that can increase handover time. In the MIH standard, MN initiates handover upon receiving RSS below the predefined threshold. The time required for handover is constant even if the MIIS server is located many hops away. When MIIS server is located many hops away, a longer time is required for $\mathrm{MN}$ to get the information of the available networks. If an alternate route is available for MIIS server, which consists of several hops in the case of route failure, then the time required for handover will also increase that may cause the breaking of connection during handover process in the worst condition. MIH standard utilize RSS for handover initiation. RSS suffered from different problems such as wrong network selection and too early and late handover, as shown in Figure 1.

Therefore, to address the challenges above, this paper introduces QoS based efficient handover scheme in which a BS collects information, in advance, of the available networks for $\mathrm{MN}$. In this scheme, MN does not wait for long time in a handover region for the collection of information of the available network. Each time when MN needs information of available networks, it is available one hop away from BS. This straightforward and distinct scheme has many advantages including the following: (i) MN search for nearby BS/AP is done in an efficient manner, (ii) the proposed scheme is not affected by the number of incoming connections, and (iii) $\mathrm{MN}$ does not consume excess energy when it scans for nearby network.

The remainder of this paper is organized as follows. In Section 2, we give a brief background of the existing schemes and their shortcomings faced by handover for M2M communications in HetMANET. In Section 3, we propose an energy efficiency vertical handover scheme from M2M communication. In Section 4, a detailed analytical and simulation analysis is discussed in detail. Finally, Section 5 offers a conclusion.

\section{Related Work}

In the last decade, various schemes have been proposed for the improvements of handover management in HetMANET. Most of these schemes are based on the optimization of different parameters necessary for handover. The optimization of these parameters reduces the handover time and latency. With the passage of time, the number of the new access networks was rapidly increased, producing signaling overhead and other issues related to handover phenomenon. Similarly, the access new technologies such as LTE Advanced and Bluetooth 4.0 low energy were introduced to save communication time and energy. All of the recent technologies try to provide its customer with the best QoS. The QoS of a network can be enhanced if a consumer is provided with a continuous connection to different networks.

To upgrade the QoS of a network, a scheme is proposed, based on optimizing parameters such as Bit Error Rate, delay, jitter, and data rate in [14]. The decision of handover is performed by using fuzzy logic and analytic hierarchy approaches. The proposed scheme receives the context information like networks related information, user preferences, and service requirements for an efficient handover process. MNs periodically check the RSS level with the current AP/BS; if the RSS drops below a particular level, then the $\mathrm{MN}$ initiates network selection phase. The network quality scoring function is defined to evaluate the QoS of a network. The network with the highest QoS is selected for the handover. The proposed scheme takes handover initiation decision on the basis of available RSS, due to which the number of false handover indications increases considerably [15].

Considering the challenges to minimize the handover number, a scheme based on self-optimizing is proposed, which extracts velocity of the MN on the basis of location information followed by the selection of an appropriate RSS level for the handover [16]. The optimization of a single parameter is not a generic solution for handover management. A complete set of handover parameters should be optimized for an efficient handover process. The efficiency of handover can be maximized if each phase of the handover process is optimized for the best performance. The $\mathrm{MIH}$ standard does not provide an optimized solution for a handover management because this standard supports only one MIIS server for all of the available access networks. In future, the number of access networks and MNs will be increased due to advancement in network access technologies. Thus, 

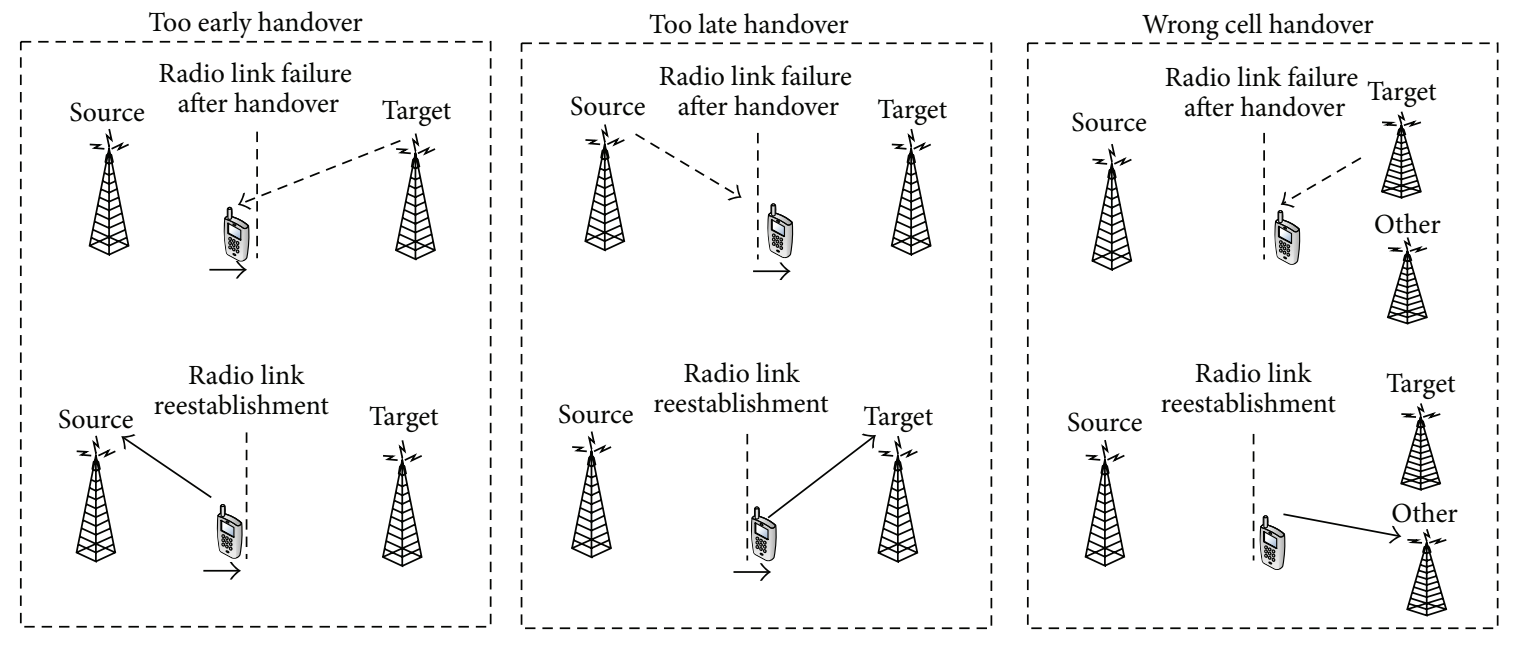

FIGURE 1: Handover problems due to RSS.

a single MIIS server will not be appropriate for all of the access networks.

In the next generation network, $\mathrm{MN}$ will be provided with multiple optimized routes to send data from one end to another. During a handover process, selection of an optimized route for data transfer is a challenging task. A scheme has been proposed to provide MN with an optimized route after the handover has been processed [12]. The proposed scheme efficiently reduced the handover latency and achieved fast recovery of the optimized path. A similar scheme has also been proposed in [13]. The scheme optimizes the route optimization for tunnel establishment to buffer packets during handover. The scheme efficiently solves buffer overflow problem in proactive handover techniques. The packet loss and handover delay due to buffer overflow are significantly minimized. However, still many issues are yet to be answered in terms of the selection of the optimal network during handover. The route optimization not only helps in balancing traffic on a particular AP/BS, but also maximizes the probability of new connections on an optimized route.

The energy consumption during the selection of networks is a major factor in a handover process in HetMANET. The energy consumption by an MN directly depends on the application running during a handover process. A scheme based on the energy efficient handover for multimedia based applications is proposed, which utilizes the concept of adapt or handover for balancing the multimedia traffic during a handover process [17]. The proposed scheme saves energy consumed due to the insignificant degradation in QoS. A single objective handover management cannot be adopted for a generic solution. However, the energy could also be reserved by the real-time power managements scheme in M2M communications, mobility management, probabilistic modeling, and graph based on M2M communications, [1821]. Furthermore, the same parameters are also achieved by optimizing data transmission in device-to-device communication and WSN, which is based on the advanced clustering scheme $[22,23]$. Such scheme is based on the received signal strength of the sensor node.
The selection of the less expensive network with best QoS during handover leads to the smooth transition of an ongoing session from one network to another. Therefore, the cost optimization must be considered during a handover process. A scheme based on cost aware handover decision is proposed which uses two cost functions, that is, triggering and priority decision [24]. Both functions are optimized for the best values of signal transmission quality, handover signaling cost, handover latency, and estimate interference. The proposed scheme efficiently transfers an ongoing session from one cell to another after checking the cost of the adjacent cells. However, still some of the parameters such as data rates and data rate based costs are not addressed in the current schemes. Therefore, we propose a solution that considers possible multiple parameters that affect the quality of a handover process in M2M.

\section{Proposed Scheme}

This section presents our proposed handover scheme for $\mathrm{M} 2 \mathrm{M}$ in detail. Figure 2 delineates the architecture of handover scheme that M2M practices. Multiple BSs and APs are deployed in the large geographic area having different networks (HetMANET), in which an MN moves from one network to another, performing some handover.

3.1. Assumptions and Definitions. In this section, we present assumptions made during the design of our network and simulation model. Some of the scenario related definitions are also given.

Assumption 1 (heterogeneous devices). All the MNs have different configuration; that is, their battery requirements are different from each other.

Assumption 2 (communication radius model). In the communication range of a BS, " $A$ " has the radius " $R$ " that is centered at " $c$." It can be defined as $\operatorname{CR}(c, R)=\{A, q \in S$ : $\left.D(A-q) \leq R_{A}\right\}$, where CR represents communication radius, 


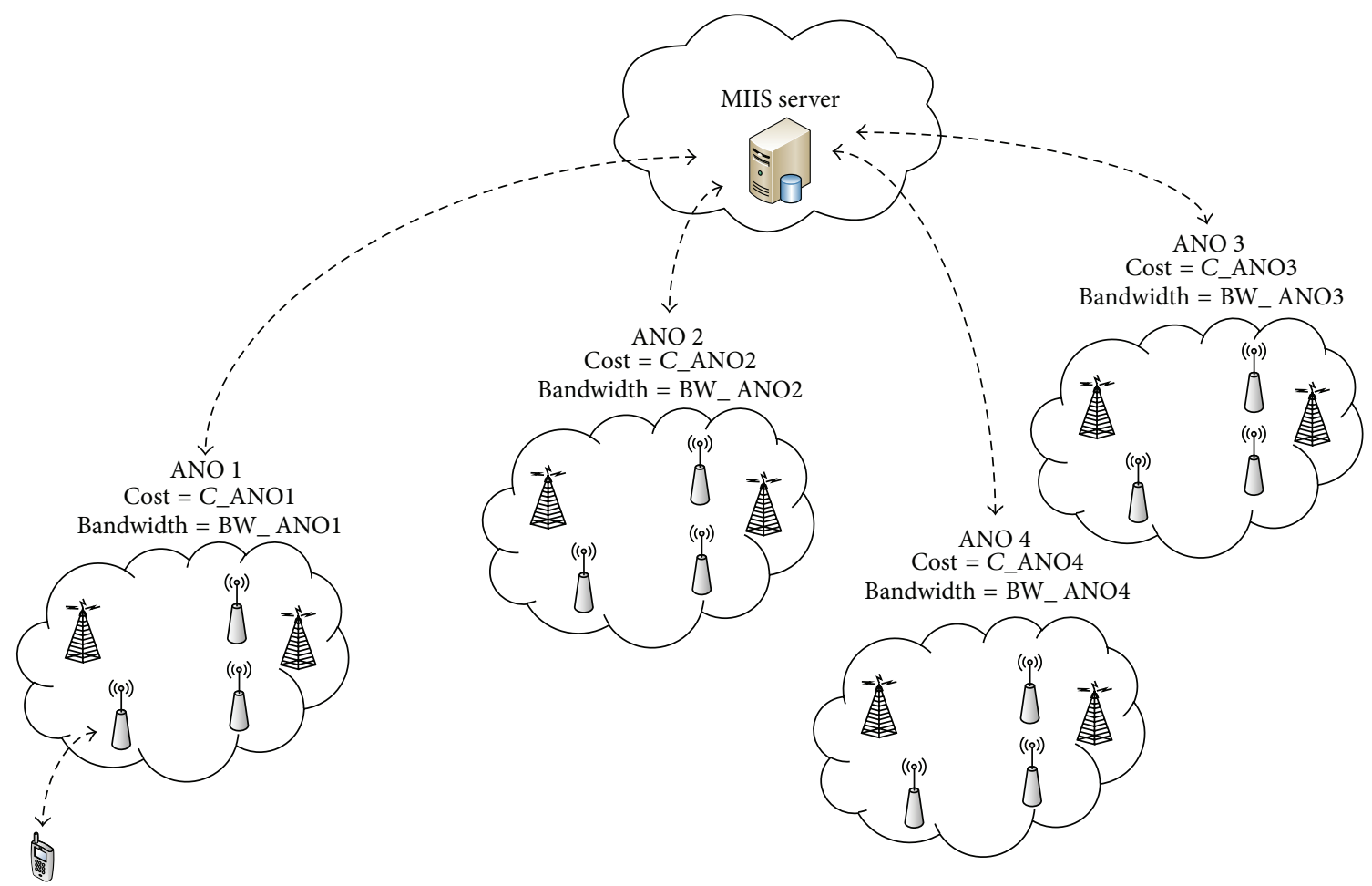

Figure 2: Proposed M2M communication handover scheme.

$S$ represents the set of deployed nodes, and $D(A-q)$ is the distance between BS and $q$ in the M2M network.

Definition 3 (Medium Scale Network). If all the MNs have direct communication access to the BS/AP, then the network is considered to be Medium Scale Network (MSN). Suppose that, in any environment, the M2M network comprising of $100 \mathrm{MNs}$ deployed in the area of $100 \mathrm{~m} \times 100 \mathrm{~m}$ is considered as MSN. This definition can be modeled as $\forall p \Lambda p \in S$, $|D(p-\mathrm{BS})|<R_{p}$, where $p$ is an $\mathrm{MN}$ among the set " $S$ " of deployed MNs and $D(p-B S)$ is the distance between any of the deployed network nodes say, $p$ and the BS. $R_{p}$ is the communication radius of node $p$.

Definition 4 (Large Scale Network). If any of the deployed $\mathrm{MN}$ does not have direct communication access to the BS/AP, then the network is considered to be Large Scale Network (LSN). Suppose that, in any environment, the M2M network comprising of $100 \mathrm{MNs}$ deployed in the area of $200 \mathrm{~m} \times$ $200 \mathrm{~m}$ is considered to be Large Scale Network (LSN). This definition can be modeled as $\exists p \Lambda p \in \mathrm{S},|D(p-\mathrm{BS})|>R_{p}$, where $p$ is a node among the set " $S$ " of deployed nodes and $D(p-\mathrm{BS})$ is the distance between any of the deployed network nodes say, $p$ and the base station. $R_{p}$ is the communication radius of node $p$.

3.2. Overview. An $\mathrm{MN}$ can perform a handover from one Access Network Operator (ANO) to another upon weak link connectivity. The MN obtains the information of cost and data rate of available networks from the MIIS server during handover to select the target network. The MIIS server stores the information of geographical locations of point of attachments (PoAs) of an ANO. Every ANO needs to send information regarding cost packages and data rates to the MIIS server. If an ANO updates either the cost model or the data rate information, it will also update this information in the MIIS server. Figure 2 delineates the fundamental idea of the proposed scheme. The proposed scheme consists of three phases: (1) handover triggering phase (2), network selection, and (3) handover execution phase.

3.2.1. Handover Triggering Phase. In the proposed scheme, we have used a threshold mechanism for handover triggering. This mean that handover is triggered if the RSS from the current network drops below a predefined threshold. An optimal threshold mechanism reduces the number of false handover indications as well as the number of handover failures to a network with overloaded APs/BSs. We set a threshold of RSS level on the boundary of the coverage area. Let $d$ represent the radius of the coverage area of AP and BS. According to the signal propagation model [25], the threshold should be set based on the distance $(1-\delta) \times d$ from $\mathrm{AP}$ or BS, where $\delta$ represents the fluctuation that is produced due to the variation in network data rate dynamics. The value of $\delta$ is taken between 0 and 1 . The threshold is given by the following equation:

$$
\theta_{T}=K_{1}-K_{2} \log ((1-\delta) d),
$$

where $K_{1}$ represents the antenna gain and signal wavelength and $K_{2}$ represents the path loss factor. Most of the traditional 


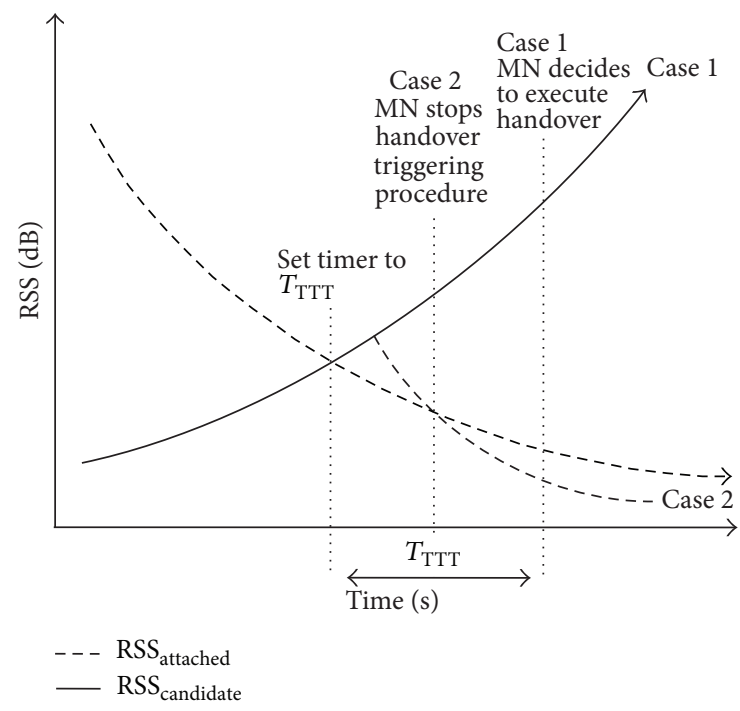

FIGURE 3: RSS based handover in M2M Communication.

approaches have used RSS for triggering handover. RSS based triggering techniques have several limitations: (i) selection of inappropriate network, (ii) an overloaded AP/BS selected for handover, and (iii) increases of false handover indications. To avoid these problems, we optimize the RSS value using (1), and it considerably reduces the false handover indications as shown as in Figure 3.

With a view to elaborating the proposed handover triggering phase, we consider a reference example based on Figure 3 [26]. In 3GPP, various handover measurements techniques are defined which supports mobility [27, 28]. However, handover triggering based on RSS and Time-toTrigger (TTT) techniques are usually used in a horizontal handover in LTE system since its simplicity and efficiency made it easy to implement [29]. As shown in Figure 3, MN periodically measures the RSS of the neighboring AP/BS. If the RSS of the candidate MN is greater than the RSS from the current $\mathrm{MN}$, the timer is set to $T_{\mathrm{TTT}} T_{\mathrm{TTT}}$ seconds by the $\mathrm{MN}$ and starts to observe RSS candidate $_{\text {and }}$ RSS ${ }_{\text {attached }}$. Apparently, if RSS candidate $>$ RSS $_{\text {attached }}$ is incessantly following during the $T_{\text {TTT }}$ seconds, the MN performs handover to the candidate MN (case 1). However, if the RSS candidate $<$ RSS $_{\text {attached }}$ follows during the $T_{\mathrm{TTT}}$ seconds, the MN stops observation and come back to its initial state (case 2).

3.2.2. Network Selection. The network selection phase is further divided into the following subsections.

(i) Cost. To select the new network for optimal handover, we need to consider the cost of applications used by an MN. The target network assigns the same cost as that of the old network to continue the movement of the MN in HetMANET. If a target network is not providing the cost that is equal to the old network, then the MN selects new cost from the target network, which is acceptable to the MN. Otherwise, the MN experiences long delay and even breaking of connection during handover. The MN normally uses different
TABle 1: Application weight table.

\begin{tabular}{lc}
\hline Application type & Weight \\
\hline Interactive & $W 1(0.1 \sim 0.3)$ \\
Elastic application & $W 2(0.3 \sim 0.5)$ \\
Voice & $W 3(0.5 \sim 0.7)$ \\
Real-time streaming & $W 4(0.7 \sim 0.9)$ \\
Demagnetizing factor & $1 \rightarrow 1 /(4 \pi)$ \\
\hline
\end{tabular}

types of applications like real-time streaming applications, voice, elastic applications (web browsing, chatting, etc.), and interactive audio and video call.

Multimedia applications require more cost as compared to elastic applications. We assign a particular weight of cost to each category of application as listed in Table 1.

Assuming that there are $M$ applications, the weights of all the applications running on the $\mathrm{MN}$, denoted by $C$, are as follows:

$$
C=\sum_{i=1}^{M} w_{i} C_{i},
$$

where $w_{i}$ represents the weight of each application. The value of various weights is taken from 0 to 1 , depending on the priority of an application. An application with the highest priority is assigned the largest weight. For instance, if MN's device is running a real-time streaming application, it will be assigned the highest weight since the streaming application can tolerate a handover delay of only $150 \mathrm{~ms}$ and a packet loss of 3\%, respectively [30]. Therefore, the MN selects a network with less possible cost, which has the potential to run a particular application during switching from one network to another.

(ii) Energy. In HetMANET, the MN consumes a significant amount of energy for scanning the available PoAs. In particular, the application with high priority needs much energy, which is normally required for fast scanning. Depending on the density of the medium (APs and BSs) of the network, the interface for a particular network is periodically switched to sleep and active states. The energy (denoted by $E$ ) required by $\mathrm{MN}$ for the scanning of a particular PoA of a network is given by

$$
E=\sum_{i=1}^{n} P_{i} \times t_{s}
$$

where $P_{i}$ is the power required by $\mathrm{MN}$ for the scanning of a PoA of an access network and $t_{s}$ represent the time taken for interface scans.

The energy required for scanning during handover depends on the application used by MN. If the request is of a high priority, then the MNs perform fast scanning. In this case, the energy required for scanning will be high. In traditional approaches, the scanning procedure is throughout the uniform and mainly depends on the RSS from an AP/BS, which leads to a high packet loss. Thus, we restrict 
the scanning energy consumption depending on the applications running by the MN during scanning. The proposed energy efficient scanning procedure significantly reduces the energy consumption by the MN during handover.

(iii) QoS Computation. The network selection phase is considered an important factor in a handover management scheme. When the $\mathrm{MN}$ is moving across the HetMANET, it performs some handover switching from one network to another. For efficient handoff, we should choose the target network that provides acceptable cost and sufficient data rate for the applications running on the $\mathrm{MN}$. Also, we need to minimize the energy for scanning a PoA for handoff. When the $\mathrm{MN}$ is moving away from the current ANO where RSS drops below a predefined threshold, it selects the target network for handoff using three metrics: cost, data rate, and energy. In our scheme, we introduce a QoS function to select an optimal network by integrating three metrics, which is given by

$$
Q=W_{c} * \ln (C)+W_{R} * \ln \left(\frac{1}{R}\right)+W_{E} * \ln (E)
$$

where $C, R$, and $E$ represent cost, data rate, and energy. Similarly, $W_{c}, W_{R}$, and $W_{E}$ are the weights of cost, data rate, and energy, respectively. The $\mathrm{MN}$ obtains the values of parameters $C$ and $R$ of available networks from MIIS server. The energy consumption by an interface using a particular application is computed by the MN. The weights are assigned randomly depending on the priority of application. This mean that the application with high data rate requires extra cost as compared to the application with fewer data. Similarly, every application requires different data rates depending on the nature of the application. For instance, real-time application requires more data rate as compared to an elastic application. Therefore, a target network is selected on the basis of cost, data rate, and energy which requires an interface of the MN during handover using (4).

Algorithm 5 (handover triggering and network selection).

(1) Computing Threshold $\theta_{\mathrm{T}}$

$$
\begin{aligned}
& \text { Get distance } d: \mathrm{MN} \longleftrightarrow \mathrm{AP} / \mathrm{BS} \\
& \text { Compute } \delta \\
& \text { Normalized distance } \leftarrow(1-\delta) \times d \\
& K_{1} \leftarrow \text { path loss factor at the edge of the cell } \\
& K_{1} \leftarrow \text { path loss factor at the distance } d \\
& \theta_{T}=K_{1}-K_{2} \log ((1-\delta) d)
\end{aligned}
$$

Get RSS at distance $d+i$,

$$
\text { where } i= \begin{cases}i+5, \text { where } i=1 & \text { if } \mathrm{PoA}=\mathrm{AP} \\ i+10, \text { where } i=1 & \text { if } \mathrm{PoA}=\mathrm{BS} .\end{cases}
$$

(2) Network Selection

$$
\begin{aligned}
& \text { Cost computation: } \\
& N_{i} \rightarrow C_{i} \rightarrow \text { MIIS server }
\end{aligned}
$$

$M \leftarrow$ Number of Applications

Assigning weight to each application

$C=\sum_{i=1}^{M} w_{i} C_{i}$

Energy computation:

$P \leftarrow$ power require by interface for scanning

$t_{s}=$ scanning slot

$E=\sum_{i=1}^{n} P_{i} \times t_{s}$

QoS Computation:

$$
Q_{i}=W_{c} * \ln \left(C_{i}\right)+W_{R} * \ln \left(\frac{1}{R_{i}}\right)+W_{E} * \ln \left(E_{i}\right)
$$

where $i=1, \ldots, n$.

(iii) Optimal AP/BS Selection. Once a particular network is selected for handover, the next step is to select an appropriate AP/BS for handover. As we know that the competition among different networks is increasing every day. Each network is trying to provide best QoS with low cost and high data rate to their user. Similarly, every network is attempting to deploy AP/BS everywhere to provide an $\mathrm{MN}$ with "always best connected" functionality. To achieve similar functionality in our scheme, we implant a handover decisionmaking model in our proposed scheme to provide an $\mathrm{MN}$ with the best AP/BS in HetMANET environment. There are several decision-making schemes available in the literature. The TOPSIS decision model has remarkable applications in handover management [31-33]. Therefore, we used TOPSIS decision-making scheme to select one of the APs/BSs for handover. There are two types of criteria available for the selection of the AP/BS. The first type directly affects the performance of an AP/BS and the second type increases the performance of the AP/BS. To minimize the imbalance effect of both of these parameters, we choose only those parameters that directly affect the performance of an AP/BS. We choose five different criteria for the selection of the network. These criteria include delay $(\alpha)$, jitter $(\beta)$, Bit Error Rate (BER) $(\gamma)$, communication cost $(c)$, and response time $(\sigma)$. The decision-making matrix $(M)$ is represented as follows:

$$
M\left(x_{i j}\right)=\left[\begin{array}{ccccc}
\alpha_{1} & \beta_{1} & \gamma_{1} & c_{1} & \sigma_{1} \\
\alpha_{2} & \beta_{2} & \gamma_{2} & c_{2} & \sigma_{2} \\
\vdots & \vdots & \vdots & \vdots & \vdots \\
\alpha_{m} & \beta_{m} & \gamma_{m} & c_{m} & \sigma_{m}
\end{array}\right]
$$

The maximum and minimum value of a parameter in a network is represented through $P_{j}^{*}=\max _{1 \leq i \leq m}\left(x_{i j}\right)$ and $P_{j}^{\circ}=\min _{1 \leq i \leq m}\left(x_{i j}\right)$, respectively, where $P$ represent a parameter. It is also important to normalize the decision-making matrix. Therefore, we perform linear scaling by checking the distance of each criterion from minimum $\left(x_{i j}^{*}=x_{i j} / P_{j}^{*}\right)$ and 
maximum $\left(x_{i j}^{\circ}=x_{i j} / P_{j}^{\circ}\right)$ values. The normalized decisionmaking matrix $\left(M^{*}\right)$ becomes as follows:

$$
M^{*}\left(x_{i j}\right)=\left[\begin{array}{ccccc}
\alpha_{1}^{*} & \beta_{1}^{*} & \gamma_{1}^{*} & c_{1}^{*} & \sigma_{1}^{*} \\
\alpha_{2}^{*} & \beta_{2}^{*} & \gamma_{2}^{*} & c_{2}^{*} & \sigma_{2}^{*} \\
\vdots & \vdots & \vdots & \vdots & \vdots \\
\alpha_{m}^{*} & \beta_{m}^{*} & \gamma_{m}^{*} & c_{m}^{*} & \sigma_{m}^{*}
\end{array}\right] .
$$

The superscript $(*)$ is used to represent criteria after normalization.

The proposed approach is purely user based decision handover scheme. Therefore, we give the user the option to assign each criterion a particular weight. These weights help us in calculating negative and positive ideal situation of a network. In particular, an AP/BS with the more positive ideal situation is closer for the selection of handover. The weighted normalized matrix $(Z)$ is represented as follows:

$$
Z=\left[\begin{array}{ccccc}
w_{1} \alpha_{1}^{*} & w_{2} \beta_{1}^{*} & w_{3} \gamma_{1}^{*} & w_{4} c_{1}^{*} & w_{5} \sigma_{1}^{*} \\
w_{1} \alpha_{2}^{*} & w_{2} \beta_{2}^{*} & w_{3} \gamma_{2}^{*} & w_{4} c_{2}^{*} & w_{5} \sigma_{2}^{*} \\
\vdots & \vdots & \vdots & \vdots & \vdots \\
w_{1} \alpha_{m}^{*} & w_{2} \beta_{m}^{*} & w_{3} \gamma_{m}^{*} & w_{4} c_{m}^{*} & w_{5} \sigma_{m}^{*}
\end{array}\right]
$$

After calculating the weighted normalized decision matrix, the next step is to compute the ideal situations, as we choose those parameters that directly affect the performance of an AP/BS. Therefore, the maximum and minimum value in each column of the matrix $Z$ is represented through negative $\left(S^{-}\right)$and positive $\left(S^{+}\right)$ideal situations, respectively, using the following relations:

$$
\begin{aligned}
& S^{+}=\left[\left(\min _{i} z_{i j} \mid j \epsilon J\right)\right]=\left[p_{1}^{*}, p_{1}^{*}, \ldots, p_{5}^{*}\right], \\
& S^{-}=\left[\left(\max _{i} z_{i j} \mid j \epsilon J\right)\right]=\left[p_{1}^{\circ}, p_{2}^{\circ}, \ldots, p_{5}^{\circ}\right] .
\end{aligned}
$$

To check whether these ideal situations fulfill the requirements of an appropriate AP/BS, we compare them with the reference ideal situation. Similarly, TOPSIS also ranks the available AP/BSs by comparing the ideal situations with reference situations. Therefore, we check the distance of each criterion from $\left(S^{-}\right)$and $\left(S^{+}\right)$using the followings relations:

$$
\begin{aligned}
& H_{i}^{+}=\sqrt{\sum_{k=1}^{5}\left(z_{i k}-p_{k}^{*}\right)^{2}}, \quad i=1,2, \ldots, n, \\
& H_{i}^{-}=\sqrt{\sum_{k=1}^{5}\left(z_{i k}-p_{k}^{\circ}\right)^{2}}, \quad i=1,2, \ldots, n,
\end{aligned}
$$

where $\mathrm{H}_{i}^{+}$and $\mathrm{H}_{i}^{-}$represent the degree of negative and positive ideal situations. To elaborate the structure of these situations, we illustrate it in Figure 4.

Finally, the optimal AP/BS is selected by computing the relative degree approach $(R)$ of each AP/BS as follows:

$$
R_{i}^{*}=\frac{H_{i}^{-}}{H_{i}^{+}+H_{i}^{-}} \text {. }
$$

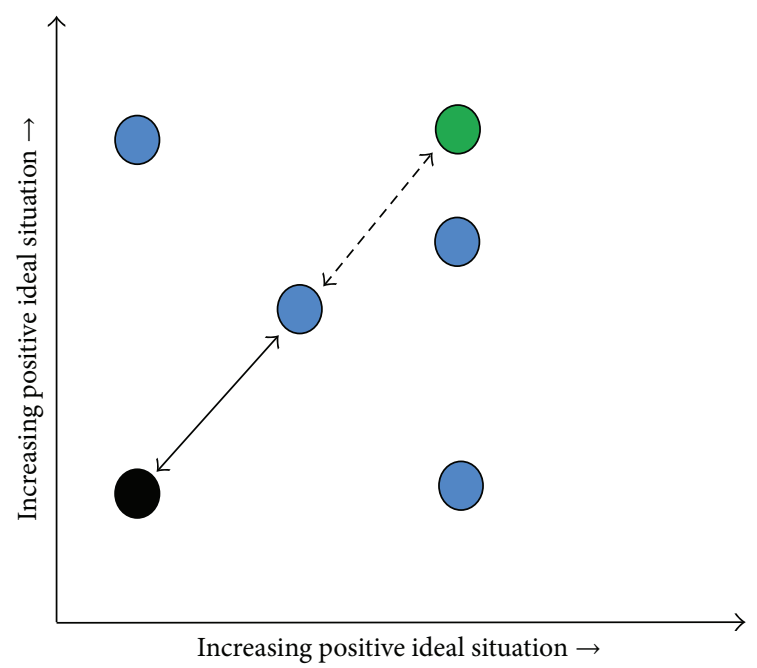

Negative ideal situation $\leftrightarrow$ Positive distance
Positive ideal situation $\longleftrightarrow$ Negative distance
Alternatives

FIGURE 4: Explanation of ideal situation.

If there are multiple AP/BSs available in a HetMANET environment, then one can compute the degrees of each $\mathrm{AP} / \mathrm{BS}$ and then sort them to select the one with the highest degree. In general, the working of the TOPSIS decision model is illustrated in Algorithm 6.

Algorithm 6 (working of TOPSIS).

(1) Initialization and normalization of matrix $M$ :

$$
M_{i j}=\frac{f_{i j}}{\sqrt{\sum_{i=1}^{k} f_{i j}^{2} i=1,2, \ldots, m ; j=1, \ldots, n}} .
$$

(2) Computing the weighted normalized matrix whose elements are

$$
Z_{i j}=w_{j} * M_{i j}, \quad i=1,2, \ldots, m ; j=1, \ldots, n .
$$

(3) To determine the ideal situations (positive and negative):

$$
\begin{aligned}
& S^{+}=\min Z_{i j} \\
& \quad j \in J \mid i=1, \ldots, n=\left[z_{i}^{+}, z_{i+1}^{+}, \ldots, z_{n}^{+}\right], \\
& S^{-}=\max Z_{i j} \\
& \quad j \in J \mid i=1, \ldots, n=\left[z_{i}^{+}, z_{i+1}^{+}, \ldots, z_{n}^{+}\right] .
\end{aligned}
$$

(4) Computing the separation measure of each situation:

$$
\begin{aligned}
& H_{i}^{+}=\sqrt{\sum_{k=1}^{n}\left(z_{k j}-z_{k}^{+}\right)^{2}}, \quad k=1,2, \ldots, m, \\
& H_{i}^{-}=\sqrt{\sum_{k=1}^{n}\left(z_{k j}-z_{k}^{-}\right)^{2}}, \quad k=1,2, \ldots, m .
\end{aligned}
$$




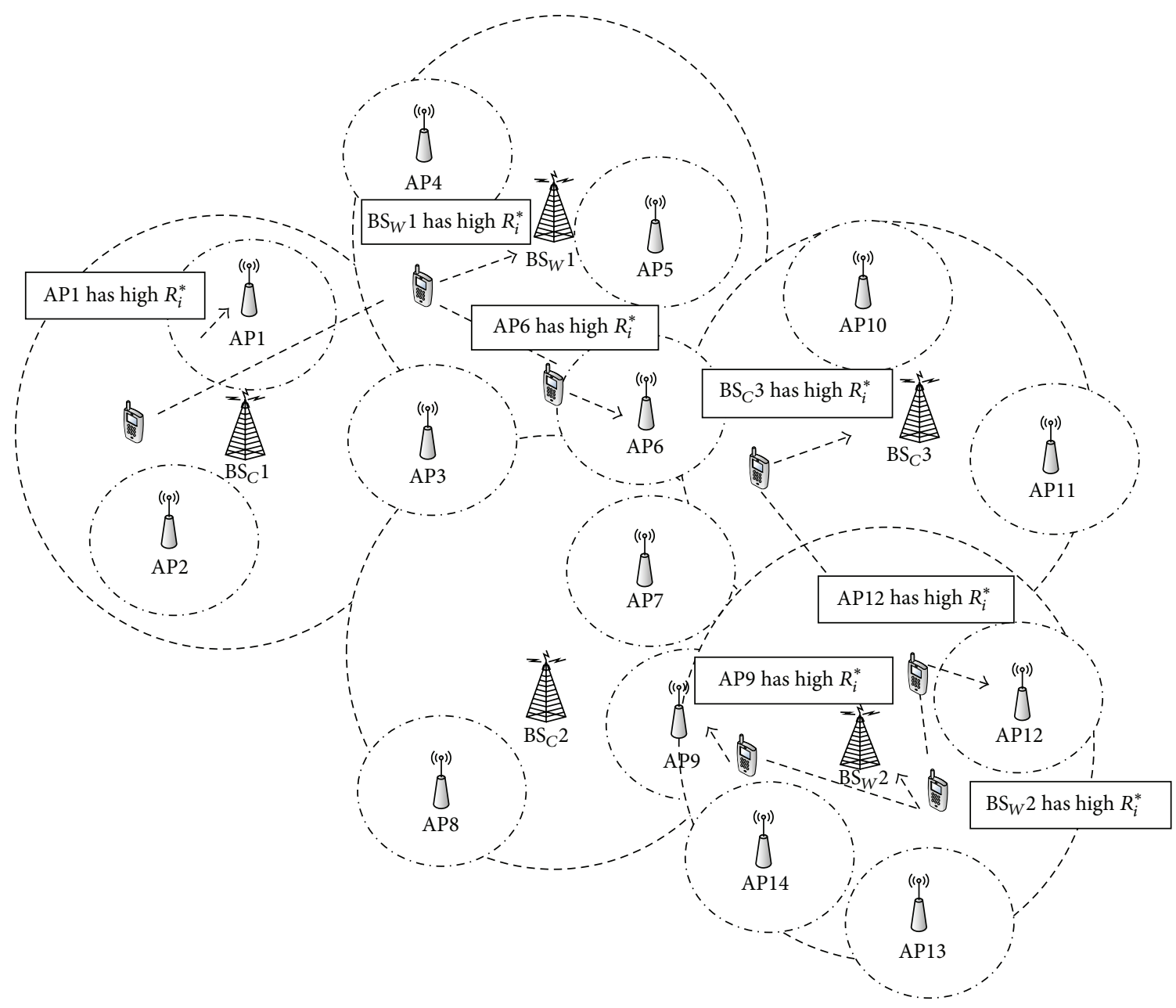

- - - MN's direction

$-\rightarrow$ MN's connection

FIGURE 5: Proposed simulation scenario.

(5) Computing relative closeness of each criterion to ideal situation.

3.2.3. Handover Execution. The $\mathrm{MN}$ performs handover execution after selecting the network with the highest QoS. The MN requests the serving AP/BS to connect the network. The AP/BS forwards this request to the MIIS server. The new network sends a connection response to the $\mathrm{MN}$, and the $\mathrm{MN}$ performs handover to the new $\mathrm{AP} / \mathrm{BS}$. The $\mathrm{MN}$ releases the resources and terminates the connection with the old network.

\section{Performance Evaluation}

In this section, we present the simulation results to highlight the benefits of the proposed handover triggering and network selection scheme. First, we show the advantages of the QoS aware network selection scheme. Second, we performed some experiments to check the handover decision model in dense and low coverage HetMANET environment. Furthermore, we evaluate the working of the proposed scheme in $\mathrm{C}$ programming language. The proposed approach is tested on three different networks, that is, WIFI, UMTS, and WiMAX. Different numbers of mobile nodes are tested in the proposed scenario with a speed ranging from 10 to $100 \mathrm{~km} / \mathrm{h}$. The number of applications is assigned randomly to each $\mathrm{MN}$ during initialization. In Figure 5, we are using only two BSs of the WiMAX network and three BSs of the cellular network because of the availability of space. In the actual simulation scenario, we used around 15 and 20 WiMAX and cellular BSs, respectively. The simulation time is set differently with the number of nodes. We test four sets of nodes, that is, 25, 50, 75 , and 100, with a simulation time of $30,60,90$, and 120 minutes, respectively. The MIH standard does not implement MIIS server in NS $2.29 \mathrm{~V} 3$. Therefore, we implement the MIIS server to store the cost and data rate information of the available networks. Moreover, the proposed scheme is tested 
for a longer duration of time to check its performance and quality in high speed and congested scenarios. The proposed $\mathrm{M} 2 \mathrm{M}$ communication scenario in HetMANET scenario is shown in Figure 5.

In Figure 5, the MN is initially connected with the AP1. After moving away from the AP1, the MN has found three different types of networks, that is, $\mathrm{BSc}, \mathrm{BSw}$, and AP3. In general, an MN has three different networks to decide handover to one of them. Our proposed approach enables the MN to scan the available networks and compute the QoS of each network. The MN found that BSw (WiMAX) provided the highest QoS. Therefore, the MNs choose WiMAX network for handover. The MN also uses the proposed decision model to check the available BSs of the WiMAX network. Similarly, the MN found the BSw1 with the highest degree and, therefore, the MN performs handover to it. Furthermore, the MN continues its movement in the proposed scenario. The handover is shown on the label attached to the MN in Figure 5.

The cost for UMTS network is fixed, and the costs for WIFI and WiMAX networks are generated randomly. We used two types of cost values for each network, that is, cost per minute and cost per data volume. The value of selection criteria for the network as well AP and BS is given in Table 2.

The energy consumption values by each interface are taken randomly from the ranges present in Table 2. Similarly, the RSS values are generated depending on the data rate. During the simulation, we periodically check the relation between data rate and RSS. When the data rate is increasing, the RSS is decreasing as they are in indirect proportion to each other.

We used five different parameters for the selection of AP/BS of the target network. As previously discussed, these parameters are indirectly eruptional to the performance of an AP/BS. The values of all of these parameters depend on the distance of $\mathrm{MN}$ and $\mathrm{AP} / \mathrm{BS}$. If the $\mathrm{MN}$ is away from the AP/BS, the values are high, and as the MNs move closer to the $\mathrm{AP} / \mathrm{BS}$, the values are decreasing. Initially, we do not set any particular values for these parameters. The values are changing with the distance of MN from the target AP/BS. Therefore, to achieve the optimal changing of these parameters, we implant a location management system using the coordinate geometry. The location management is simulated, and we obtain remarkable results. Finally, we do not simulate the handover execution phase and left this to the network operator.

An interface requires high energy if the $\mathrm{MN}$ is running an application that requires higher data rate. For example, the streaming application requires higher data rate compared to the elastic application. Therefore, a streaming application consumes more energy than an elastic application. Similarly, the MN consumes more energy on scanning if the numbers of available networks are high. Sometimes, the MN consumes unnecessary energy on scanning available networks that are far away from the MN. Therefore, in the proposed approach, we perform a dynamic sort of scanning based on the density (number of APs and BSs) of the medium. The results obtained from simulating the density based scanning are shown in Figure 6. The result shows that the energy consumption
TABLE 2: Simulation values in the proposed scheme.

\begin{tabular}{lcc}
\hline Column heading & Column heading two & Column heading three \\
\hline Data rate (kbps) & $0-1200$ & $0-1500$ \\
Energy (W) & 4.0 & 3.0 \\
Cost & $0-30$ & $10-50$ \\
RSS (dB) & $1-20$ & $1-20$ \\
\hline
\end{tabular}

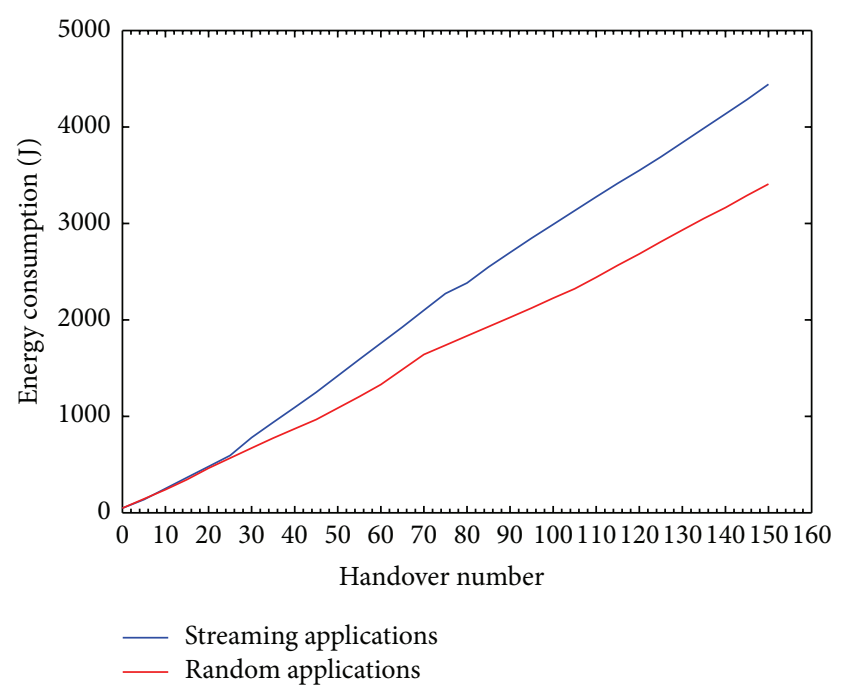

FIGURE 6: Application based energy consumption.

is significantly optimized by scanning a particular set of APs and BSs. Similarly, the energy that is consumed on unnecessary scanning is highly reduced. The performance of the proposed scheme shows that the energy consumed on scanning of all of the available AP/BSs of all networks is now reduced to one particular network.

We compute the device lifetime by running the simulation for a longer duration of time with the different application running on the MN's device. The $\mathrm{MN}$ is assigned periodically various applications. Similarly, the MN performed several handover ranging from hundreds to thousands. The device lifetime is recorded in both with and without proposed schemes. The efficient selection of the target network and AP/BS highly reduced the energy required for scanning. Therefore, the device lifetime is also increased. As shown in Figure 7, the device is consuming two types of energies (1) on Scanning and (2) running different applications.

We evaluate the energy consumption of the MN against the scanning number of APs/BSs. The MN scans a particular set of APs/BSs. Therefore, it requires less amount of energy. In Figure 8, we compared the performance of the proposed energy optimization with the scheme presented in [34]. We compute the scanning time of an interface against the number of APs/BSs. Furthermore, we compute the average energy consumption by an application using all of the three interfaces. Finally, the results are calculated and drawn in Figure 8. If an application used by the MN has high priority, then the MN needs fast scanning, which requires higher energy as compared to the application with lower priority. 


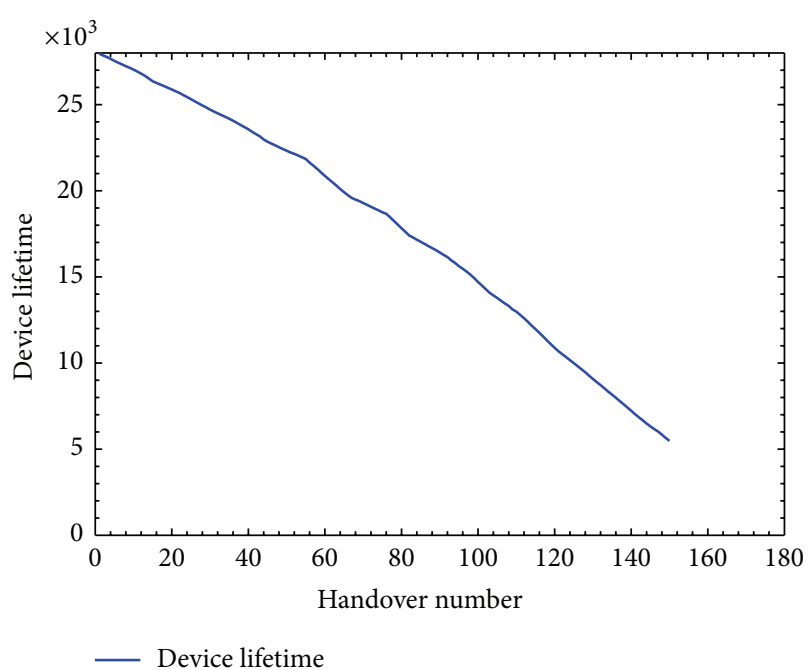

FIgURE 7: Device lifetime.

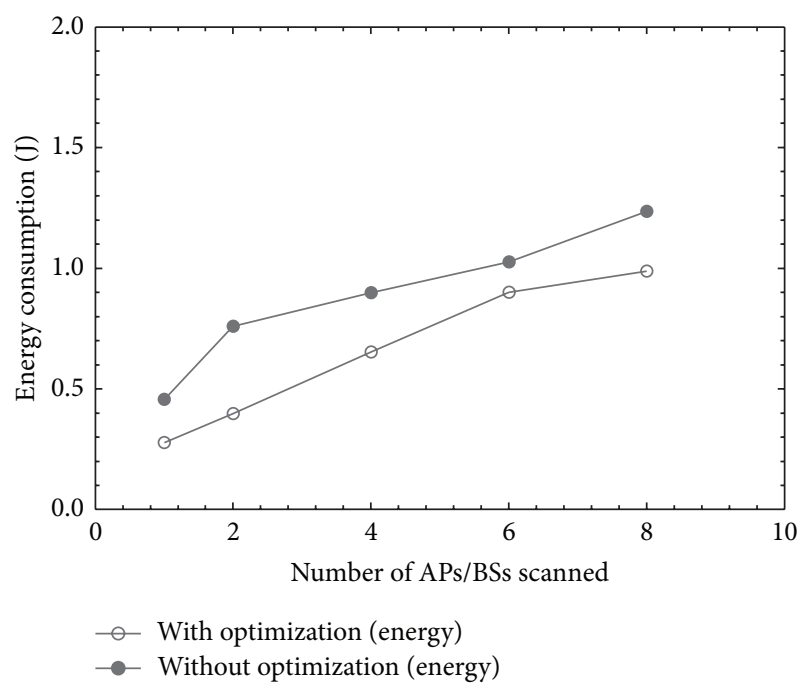

FIGURE 8: Performance analysis of energy optimization.

Therefore, we assign the $\mathrm{MN}$ the highest weight if the applications running on it have high priority and vice versa. The range of weights for energy is taken from 0 to 1.0. The proposed optimization of energy required less energy than the existing scheme due to the new power aware interface management scheme.

The AP/BS can provide services to a limited number of MNs. As the number of MNs increases on AP/BS, the QoS decreases. Therefore, we need traffic management that can efficiently balance the number of connections on a particular AP/BS. If the number of connections on $\mathrm{AP} / \mathrm{BS}$ exceeds a given threshold $(\varphi)$, the AP/BS should not accept any more connections. If the $\mathrm{MN}$ requests a connection to an overloaded AP/BS, the AP/BS rejects the connection application for the particular MN. To address the issue above,

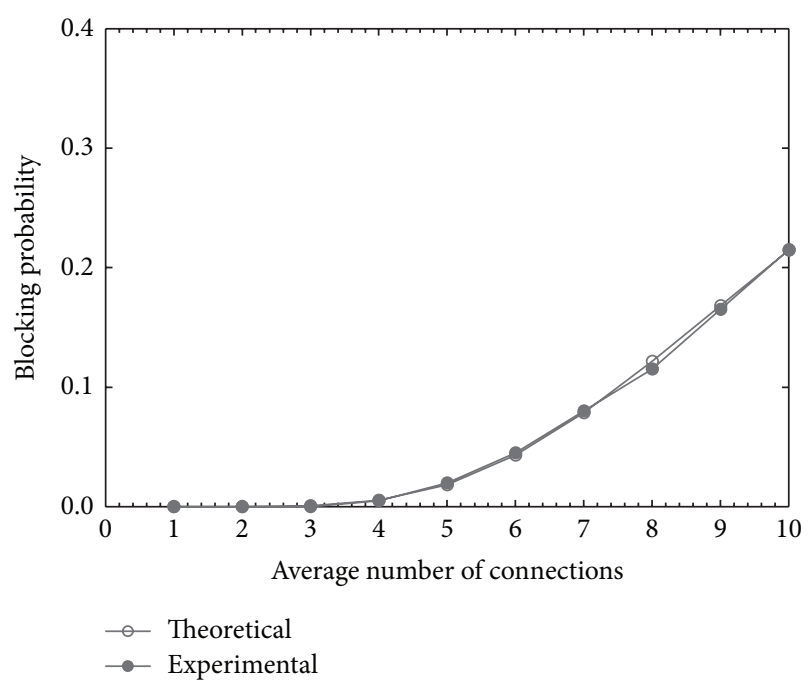

FIGURE 9: Theoretical and experimental blocking probability.

we modeled the traffic on an AP/BS. Let $\lambda$ be the number of connections on AP/BS computed as follows:

$$
\lambda=\lambda+\sum_{i=1}^{N} \tau_{i} \quad \text { where } \lambda<\varphi,
$$

where $\gamma$ represents the number of connections already present on an AP/BS and the $\tau$ is a new connection arriving on AP/BS. We define a two-state Markov chain model for $\mathrm{AP} / \mathrm{BS}$. In the first state, the AP/BS accepts new connection since it has vacant channels available for new connections, while in the second state the AP/BS does not accept new connections since it has no available channels for incoming connections. We called the first state as an open state, whereas the other one is a close state. The probability of a close and open state depends on the leaving and joining of new MNs, respectively.

If an AP/BS reaches a close state, then it blocks any incoming connections. The probability of blocking a new connection can be represented as follows [24, 35]:

$$
P_{b}=\sum_{i=0}^{C}\left(1-\beta_{i+1}\right) \times P_{i},
$$

where $P_{i}$ is the probability of a channel that is either busy or available and $\beta$ is the state of an AP/BS whether it is in the open or closed state. We restrict the boundaries of $\beta$ to either 1 (open state) or 0 (close state). The computation of the blocking probability on an AP/BS is summarized in Algorithm 7.

We performed theoretical and experimental analysis of the proposed handover blocking probability results in Figures 9 and 10. As shown in Figure 9, theoretical and experimental results are almost similar demonstrating that increasing the number of new connections consequently increases the blocking probability. Similarly, in Figure 10, the theoretical and experimental analysis is giving similar results, with the increase in a mean number of connections, affecting the total 


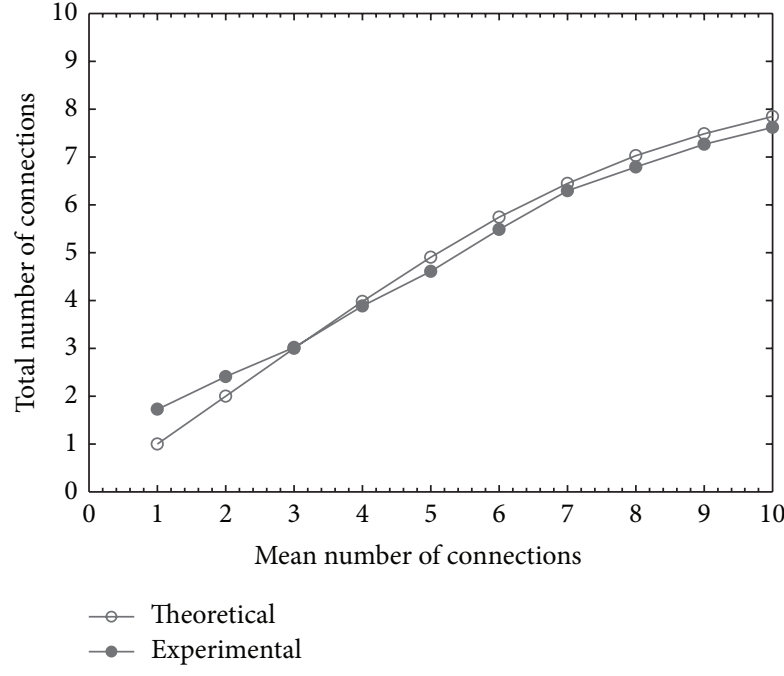

Figure 10: Mean number of connections.

number of connections. The experimental analysis has been carried in "C programming" language, while the numbers of MNs are distributed randomly using Poisson distribution.

Algorithm 7 (computation of blocking probability).

$M \leftarrow$ Maximum number of connections

$$
\begin{aligned}
& \varphi \leftarrow \text { Connections threshold } \\
& P \leftarrow \text { Probability of busy or available state } \\
& P_{b} \leftarrow \text { blocking probability } \\
& \beta \leftarrow 0 \text { or } 1 \text { (close or open state) }
\end{aligned}
$$

While $(\lambda<\varphi)$ and (index $\leq M)$ do

$$
\begin{aligned}
& \lambda=\lambda+\sum_{i=1}^{N} \tau_{i} ; \\
& \text { if }(\lambda>\varphi) \\
& P_{b}=\sum_{i=0}^{C}\left(1-\beta_{i+1}\right) \times P_{i} \\
& \text { if }\left(P_{b}==1\right)
\end{aligned}
$$

block new connections

else

accept new connection

index++

end while

The experimental and theoretical results are very close to each other which shows the accuracy of the proposed approach. The employing of blocking probability approach significantly models the traffic on an AP/BS. Moreover, the MNs do not scan those APs/BSs, which are already in a close state. Thus, using this way, the proposed approach always provides the MN with only those APs/BSs, which has channels available for the new connections.

Similarly, we test every possible probability of incoming connection on AP/BS. The probabilities are divided into three

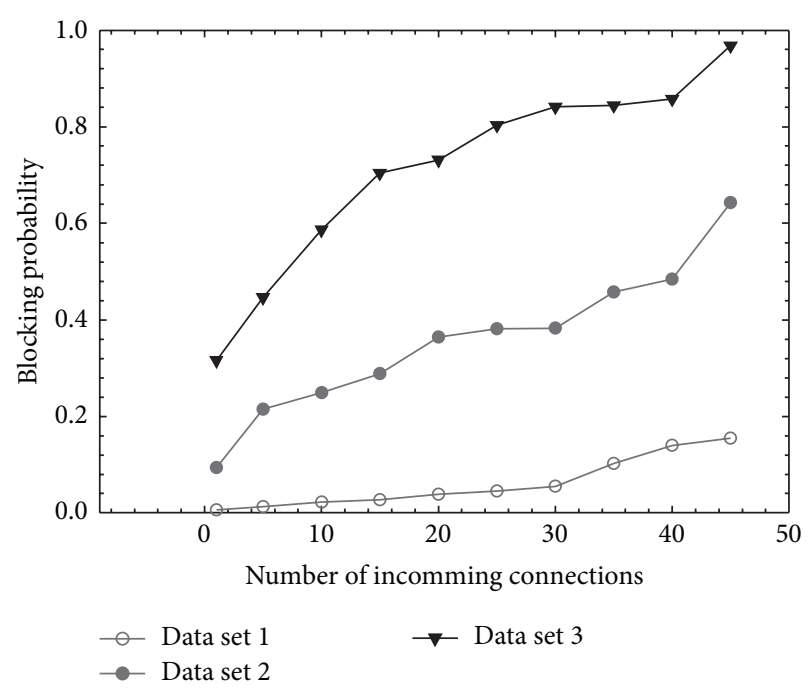

FIGURE 11: Performance analysis of traffic management.

different data sets; that is, the first set is $0.1 \sim 0.3$, the second set is $0.4 \sim 0.7$, and the third set contains $0.8 \sim 1.0$ with high probability of close state in data set 3 , average close state probability in data set 2 , and, similarly, low probability in data set 1 . On each channel of an AP/BS, we test all the possible probabilities from set 1. Furthermore, we compute the average blocking probability of AP/BS in the meantime when there is no particular channel available for incoming connection, or it is already in use. In Figure 11, we have shown that the blocking probability is high for data set 3 since most of the channels are already occupied. Similarly, for 1 and 2 data sets, the close state is considerably small as compared to the first data set. It is also shown that the increase in the number of incoming connections increases the blocking probability. In fact, upon arrival of new connections on the AP/BS, the channels are occupied and are switched to the close state. The close state probability is evaluated by using the following equation:

$$
P_{\text {Close }}=\frac{P_{C} \times P_{N}}{1-P_{H} \times\left(1-P_{C}\right)},
$$

where $P_{C}, P_{H}$, and $P_{N}$ are the probabilities of a channel availability or unavailability, holding time distribution, and new call attempt that has been accepted, respectively.

Moreover, we investigated the handover initiation process on different distances from a target network. We gradually increase the velocity of an $\mathrm{MN}$ to check the variation in data rate dynamics. Each application has assigned a particular weight based on data rate requirements. The range of weights is taken from 0 to 1 . An application that requires high data rate is assigned high weight (nearly equal to 1.0) and the application which requires less data rate is assigned (nearly equal to 0 ) lower weight. Table 2 shows the different values used in the performance evaluation of data rate optimization phase.

The velocity of an $\mathrm{MN}$ is checked against the application's weight. The proposed scheme performed efficiently since both the velocity of the $\mathrm{MN}$ and the weight of the application 
TABLE 3: Simulation parameters used in data rate optimization.

\begin{tabular}{cccc}
\hline$\sigma$ & $\begin{array}{c}\text { Distance from } \\
\text { new network }\end{array}$ & $\begin{array}{c}\text { Data rate in } \\
\text { current } \\
\text { network (Mbit/s) }\end{array}$ & $\begin{array}{c}\text { Velocity of MN } \\
(\mathrm{km} / \mathrm{h})\end{array}$ \\
\hline 0.1 & 80 & 2 & 10 \\
0.2 & 75 & 2.5 & 15 \\
0.3 & 70 & 3 & 20 \\
0.4 & 65 & 3.5 & 25 \\
0.5 & 80 & 2 & 10 \\
\hline
\end{tabular}

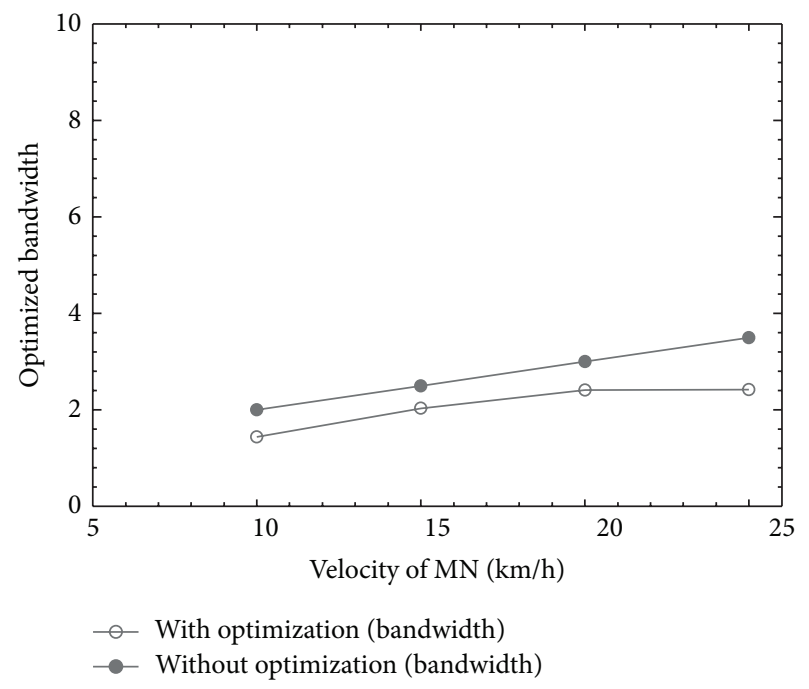

FIGURE 12: Performance analysis of data rate optimization.

are increased. It is due to the optimization of parameters used in Table 3 above. In Figure 12, the proposed with optimization data rate is employed against the without optimization data rate. The proposed optimization efficiently solves the high MN's velocity problem. The velocity of the $\mathrm{MN}$ is gradually increased, and we observed that the application that requires high data rate was shifted to the optimized data rate. Our proposed solution significantly optimized the data rate problem for the MN during the handover process.

Finally, we compute the quality of each network available in the vicinity of the MN's on the current AP/BS. The MN computes the QoS of each network using (4). The MN selects the network with the highest QoS and proceeds in contact with it. Figure 13 delineates the performance selection of an optimal network using different weights of data rate, cost, and energy ranging from 0 to 1 . The different values obtained in the performance evaluation Sections 4.1, 4.2, and 4.3 are classified into three different data sets on the basis of weights assigned to data rate, cost, and energy. In data set 1, the weight of the data rate is less compared to the cost and energy, data set 2 has less weight of energy compared to the data rate and cost, and, in data set 3 , the weight of the cost is smaller compared to the data rate and energy. The QoS of a network is tested against the user preference (random selection of applications from Table 1) in terms of the cost, data rate, and energy required during a handover process. The selection of

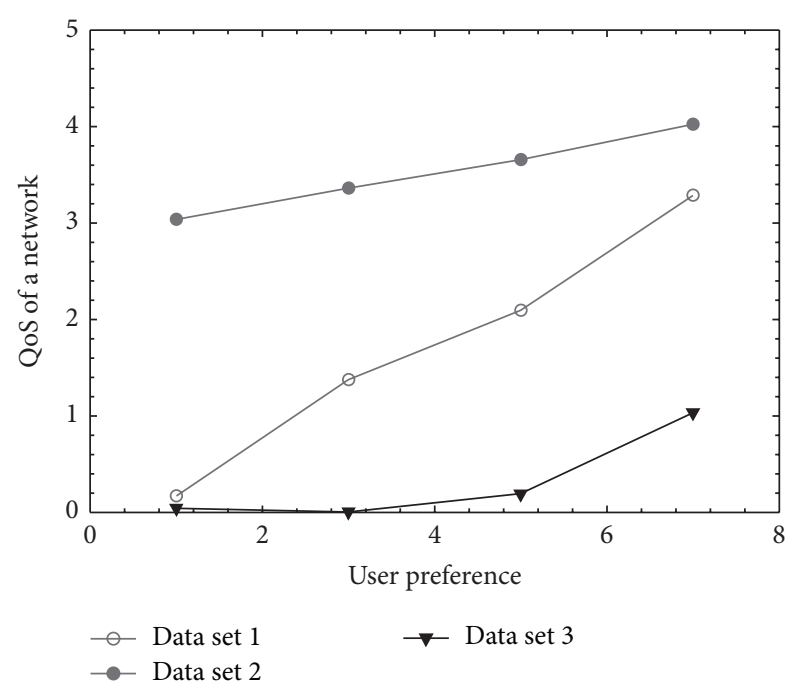

FIGURE 13: Optimization of QoS of a network.

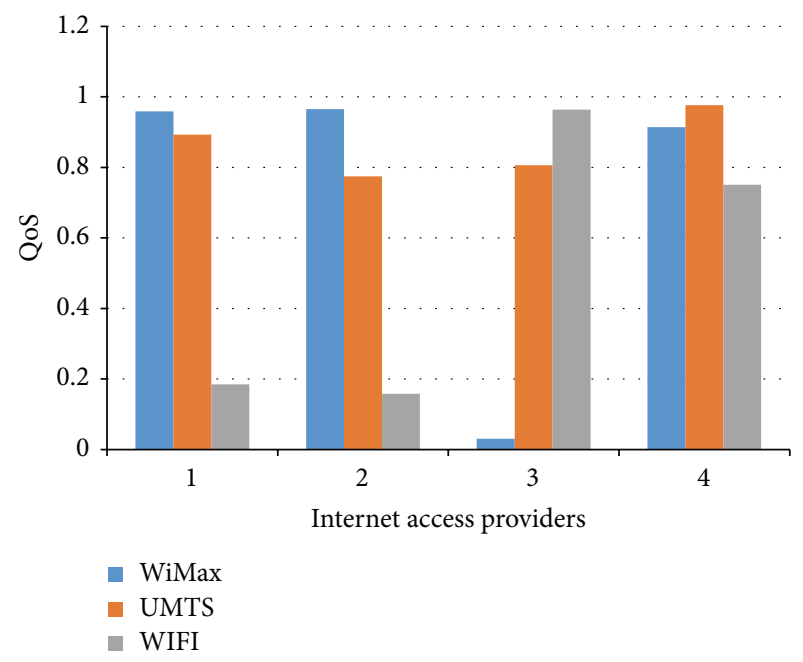

FIGURE 14: QoS during handover.

QoS of a network significantly achieves good results in the case of data set 3, which shows that most of the users preferred data rate compared to the cost and energy.

Moreover, we also compute the QoS of each network after each handover. The comparison of the technologies against the QoS is illustrated in Figure 14. The proposed scheme selects the available networks on the basis of the applications running on the MN's device.

\section{Conclusion}

In this paper, we proposed a QoS based vertical handover scheme for M2M communications in HetMANET, which represents multiparameters optimization technique for a handover process. The proposed scheme efficiently obtained the information of the communication cost of all the available networks. The scanning of the available network is performed based on the density of the AP/BS. Moreover, 
the MN optimized the energy required by an interface for scanning and making a connection to the new network. The $\mathrm{AP} / \mathrm{BS}$ optimized the traffic on AP/BS for providing the best connectivity and QoS to the users. The handover initiation phase is triggered by using the proposed optimal threshold scheme due to which the numbers of failed handover are significantly minimized. The optimizations of parameters above are quantified in a QoS function network. The QoS function returns the suitable network against the application used by the $\mathrm{MN}$ during handover. The quantitative analysis shows the accuracy and strength of the proposed scheme. For future work, we are planning to develop an optimization technique based on the decision modeling as well as fuzzy logic technique.

\section{Conflict of Interests}

The authors declare that there is no conflict of interests regarding the publication of this paper.

\section{Acknowledgments}

This work was supported by the IT R\&D Program of MSIP/IITP (10041145, Self-Organized Software Platform (SoSp) for Welfare Devices), and it was supported by the Brain Korea 21 Plus Project (SW Human Resource Development Program for Supporting Smart Life) funded by the Ministry of Education, School of Computer Science and Engineering, Kyungpook National University, Korea (21A20131600005).

\section{References}

[1] R.-H. Gau and C.-P. Cheng, "Optimal tree pruning for location update in machine-to-machine communications," IEEE Transactions on Wireless Communications, vol. 12, no. 6, pp. 26202632, 2013.

[2] M. Zorzi, A. Gluhak, S. Lange, and A. Bassi, "From today's intranet of things to a future Internet of things: a wireless- and mobility-related view," IEEE Wireless Communications, vol. 17, no. 6, pp. 44-51, 2010.

[3] Study on facilitating machine to machine communications in 3GPP systems, 3GPP TR 22.868.

[4] 3GPP, "System improvements for machine-type communications," 3GPP TR 23.888, V1.0.0, 2010.

[5] Service requirements for machine-type communications, 3GPP TS 22.368, V10.2.0, 2010.

[6] J. Kellokoski, J. Koskinen, R. Nyrhinen, and T. Hamalainen, "Efficient handovers for machine-to-machine communications between IEEE 802.11 and 3GPP evolved packed core networks," in Proceedings of the IEEE International Conference on Green Computing and Communications (GreenCom '12), pp. 722-725, IEEE, Besançon, France, November 2012.

[7] IEEE Standard for Local and Metropolitan Area Networks- Part 21: Media Independent Handover, IEEE Standard, 2008.

[8] S. Thomson and T. Narten, "IPv6 stateless address autoconfiguration," IETF, RFC 2462, 1998.

[9] H. Lin and H. Labiod, "Hybrid handover optimization for multiple mobile routers-based multihomed NEMO networks," in Proceedings of the IEEE International Conference on Pervasive Services (ICPS '07), pp. 136-144, Istanbul, Turkey, July 2007.

[10] M. I. Sanchez, M. Urueña, A. De La Oliva, J. A. Hernandez, and C. J. Bernardos, "On providing mobility management in WOBANs: integration with PMIPv6 and MIH," IEEE Communications Magazine, vol. 51, no. 10, pp. 172-181, 2013.

[11] F. Buiati, L. J. G. Villalba, D. Corujo, J. Soares, S. Sargento, and R. L. Aguiar, "Hierarchical neighbor discovery scheme for handover optimization," IEEE Communications Letters, vol. 14, no. 11, pp. 1020-1022, 2010.

[12] J. S. Choi, B. G. Jung, and T.-I. Kim, "LMA initiated route optimization protocol for improving PMIP handover performance," IEEE Communications Letters, vol. 13, no. 11, pp. 871-873, 2009.

[13] W. Yumei, F. Yufei, and Z. Lin, "Coordinating fast handover and route optimization in proxy mobile IPv6," in Proceedings of the 5th International Conference on Wireless Communications, Networking and Mobile Computing (WiCOM '09), pp. 1-4, IEEE, Beijing, China, September 2009.

[14] M. Zekri, B. Jouaber, and D. Zeghlache, "Context aware vertical handover decision making in heterogeneous wireless networks," in Proceedings of the 35th Annual IEEE Conference on Local Computer Networks (LCN'10), pp. 764-768, IEEE, Denver, Colo, USA, October 2010.

[15] P. P. Bhattacharya and P. K. Banerjee, "Signal to interference ratio based fuzzy logic controlled call handover," in Proceedings of the Annual IEEE India Conference, pp. 1-5, IEEE, New Delhi, India, September 2006.

[16] Y. Yang, P. Yu, and W. Li, "Handover self-optimization mechanism based on velocity for cellular networks," in Proceedings of the 7th International ICST Conference on Communications and Networking in China (CHINACOM '12), pp. 606-610, IEEE, August 2012.

[17] R. Trestian, O. Ormond, and G.-M. Muntean, "Energy-qualitycost tradeoff in a multimedia-based heterogeneous wireless network environment," IEEE Transactions on Broadcasting, vol. 59, no. 2, pp. 340-357, 2013.

[18] A. Paul, "Real-time power management for embedded $\mathrm{m} 2 \mathrm{~m}$ using intelligent learning methods," ACM Transactions on Embedded Computing Systems, vol. 13, article 148, 2014.

[19] A. Ahmad, S. Jabbar, A. Paul, and S. Rho, "Mobility aware energy efficient congestion control in mobile wireless sensor network," International Journal of Distributed Sensor Networks, vol. 2014, Article ID 530416, 13 pages, 2014.

[20] A. Paul and S. Rho, "Probabilistic model for M2M in IoT networking and communication," Telecommunication Systems, 2015.

[21] A. Paul, "Graph based M2M for IoT environment," in Proceedings of the Research in Adaptive and Convergent Systems (RACS '13), pp. 45-46, ACM, Montreal, Canada, October 2013.

[22] A. Ahmad, A. Dainel, and P. Anand, "Optimized data transmission using cooperative devices in clustered D2D communication," in Proceedings of the Conference on Research in Adaptive and Convergent Systems (RACS '14), ACM, Towson, Md, USA, October 2014.

[23] A. Ahmad, M. M. Rathore, A. Paul, and B.-W. Chen, "Data transmission scheme using mobile sink in static wireless sensor network," Journal of Sensors, vol. 2015, Article ID 279304, 8 pages, 2015.

[24] T. Wu, J. Huang, X. Yu, X. Qu, and Y. Wang, "Cost-aware handover decision algorithm for cooperative cellular relaying networks," in Proceedings of the 67th Vehicular Technology 
Conference (VTC '08), pp. 2446-2450, IEEE, Singapore, May 2008.

[25] Q.-T. Nguyen-Vuong, N. Agoulmine, and Y. Ghamri-Doudane, "A user-centric and context-aware solution to interface management and access network selection in heterogeneous wireless environments," Computer Networks, vol. 52, no. 18, pp. 3358$3372,2008$.

[26] S. J. Bae, M. Y. Chung, and J. So, "Handover triggering mechanism based on IEEE 802.21 in heterogeneous networks with LTE and WLAN," in Proceedings of the International Conference on Information Networking (ICOIN '11), pp. 399-403, IEEE, Barcelona, Spain, January 2011.

[27] 3GPP, "EUTRA radio resource control," 3GPP TS 36.331 V9.2.0, 3GPP, 2010.

[28] 3GPP, "Service Requirements for Machine-Type Communications," 3GPP TS 22.368 V11.0.0, 2010.

[29] D. Aziz and R. Sigle, "Improvement of LTE handover performance through interference coordination," in Proceedings of the 69th IEEE Vehicular Technology Conference (VTC Spring '09), pp. 1-5, IEEE, Barcelona, Spain, April 2009.

[30] ITU, 2014, http://www.itu.int/pub/R-QUE-SG07.

[31] E. Patouni, N. Alonistioti, and L. Merakos, "Modeling and performance evaluation of reconfiguration decision making in heterogeneous radio network environments," IEEE Transactions on Vehicular Technology, vol. 59, no. 4, pp. 1887-1900, 2010.

[32] A. Ahmed, L. M. Boulahia, and D. Gaïti, "Enabling vertical handover decisions in heterogeneous wireless networks: a stateof-the-art and a classification," IEEE Communications Surveys \& Tutorials, vol. 16, no. 2, pp. 776-811, 2014.

[33] H.-H. Choi, "An optimal handover decision for throughput enhancement," IEEE Communications Letters, vol. 14, no. 9, pp. 851-853, 2010.

[34] G. Kuhn, J. Eisl, and H. Becker, "Co-operative handover in 3G system architecture evolution," in Proceedings of the 32nd IEEE Conference on Local Computer Networks (LCN '07), pp. 643650, IEEE, Dublin, Ireland, October 2007.

[35] R. Ramjee, R. Nagarajan, and D. Towsley, "On optimal call admission control in cellular networks," in Proceedings of the IEEE 15th Annual Joint Conference of the IEEE Computer Societies. Networking the Next Generation, vol. 1, pp. 43-50, San Francisco, Calif, USA, March 1996. 

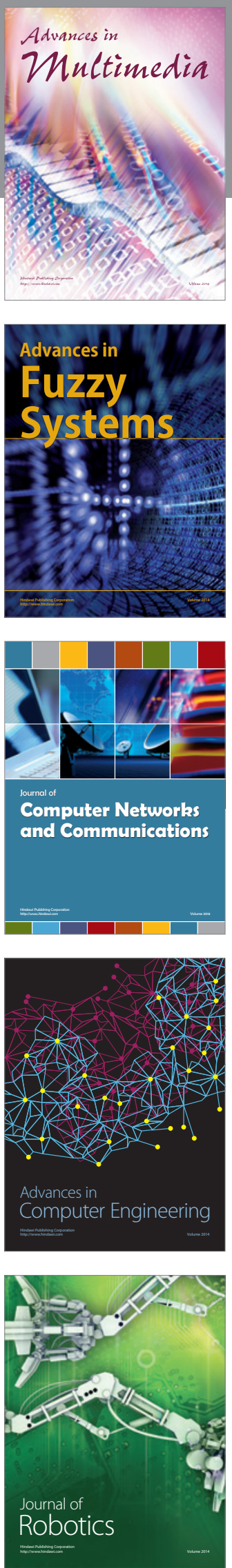

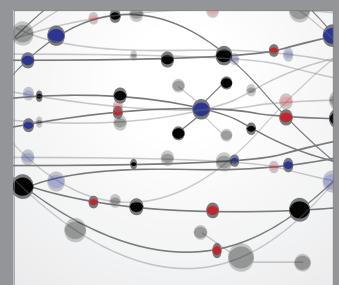

The Scientific World Journal
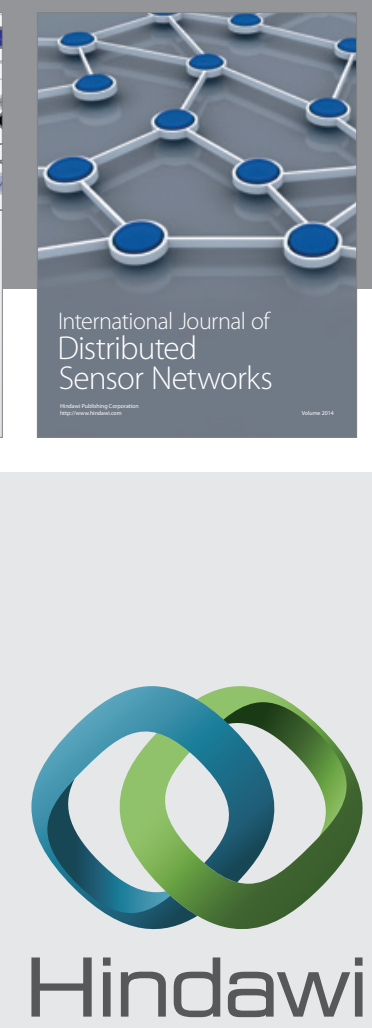

Submit your manuscripts at

http://www.hindawi.com
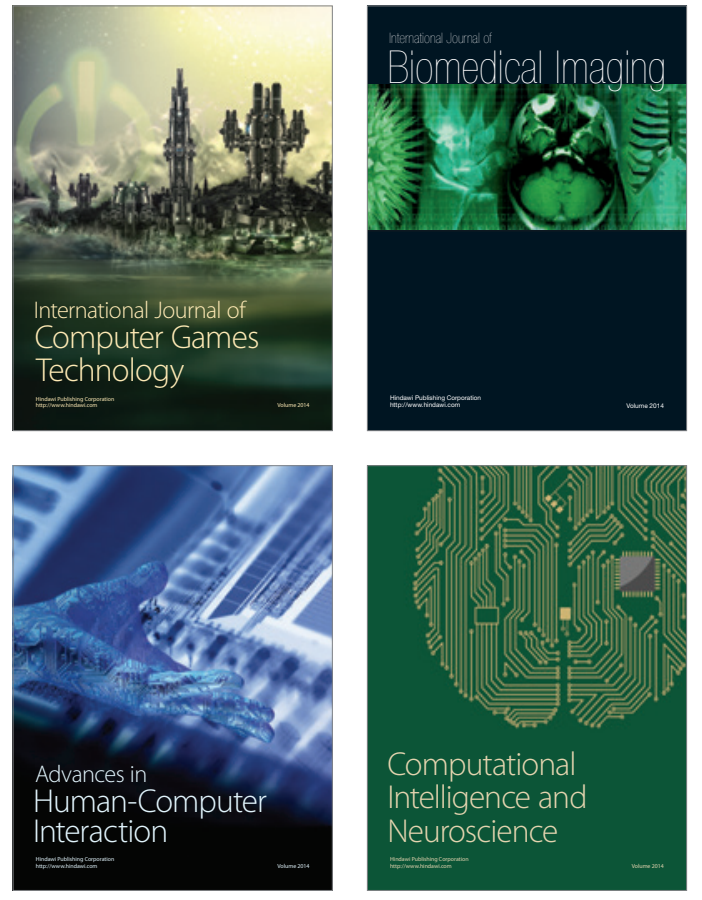
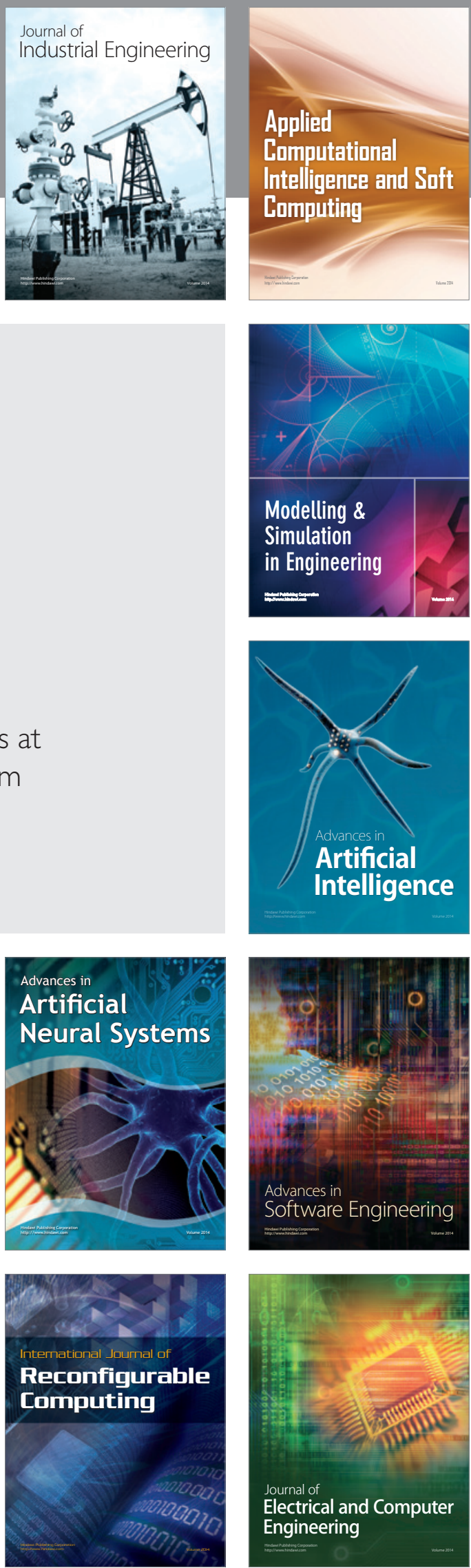